\title{
Eau et changement climatique : quelles stratégies d'adaptation pour la gestion de l'eau d'irrigation dans le sud-est Tunisien
}

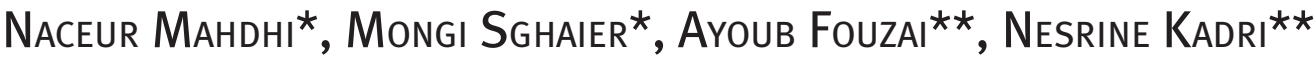

DOI: $10.30682 / \mathrm{nm} 1901 \mathrm{~b}$

Jel codes: Q25, Q54

\begin{abstract}
The objective of this study was to identify the different irrigators'strategies and to analyze the economic profitability of irrigated production systems in relation to adaptations to the scarcity of groundwater resources in a context of climate change in the South east of Tunisian. Based on surveys of 190 irrigated farms, this research shows that eighty-five percent (85\%) of respondents clearly perceive climate change in southeastern Tunisia. These changes result in a decrease and an increasing irregularity of the rains, a disruption of the winter season, a greater frequency of pockets of drought and the decrease of the piezometric level of the water tables. In response to these changes, irrigators have adopted adaptation strategies, the most common of which are: offensive strategy or "chasing", defensive strategy and contractive strategy. These strategies include various adaptation measures such as annual cleaning and deepening of wells, the use of water saving, the change of the cropping system and the reduction of irrigated area. These readjustments for most of the measures adopted by the irrigators have an impact on the elements of their operating account. In addition, offensive and defensive strategies appear to be the most economically profitable types of adaptation at the 1\% level. Knowledge of different adaptation strategies and their economic returns enables adaptation options to be identified that are both realistic in terms of implementation and ambitious in terms of their objectives, and help develop priorities for adaptation of water resources for irrigation.
\end{abstract}

Keywords: Adaptation strategy, Climate change, Irrigated perimeter, Economic profitability, Southeastern Tunisia.

\section{Introduction}

Dans un contexte de raréfaction et des risques de changements climatiques, la gestion de la ressource en eau est devenue une préoccupation majeure du monde entier. Particulièrement concernée, la Tunisie se place dans la catégorie des pays le moins dotés en ressources en eau et parmi les pays les plus sévèrement touchées par les chan- gements climatiques (MAE et GIZ, 2011 ; Nefzi, 2012 ; MEE, 2013) dans le bassin méditerranéen. Aujourd'hui l'ampleur des enjeux des changements climatiques sur l'agriculture sont inévitables et soulèvent la question de l'adaptation, qui constitue une urgence pour le secteur irrigué et notamment dans le sud-est Tunisien. Plusieurs études nationales et régionales soulignent les risques de baisse importante des ressources en

* Institut des Régions Arides de Médenine (IRA), Tunisie.

** Ecole Supérieure d'Agriculture de Mograne, Tunisie.

Corresponding author: naceur.mahdhi@ira.rnrt.tn 
eaux $(-28 \%$ pour les ressources en eaux souterraines et $-5 \%$ pour les eaux de surface), et de la dégradation de la qualité de l'eau d'irrigation (salinité plus élevée, particulièrement dans les zones côtières et le Sud tunisien) (MEDD/DGEQV, 2008 ; MAE et GIZ, 2011 ; MEE, 2013).

L'adaptation au changement climatique a reçu une attention accrue dans le débat scientifique et politique et est de plus en plus un sujet d'intérêt tant pour les producteurs que pour les décideurs (GIEC, 2001 ; Pierre et al., 2012). Elle est définie comme l'ensemble des mesures et ajustements mis en œuvre par des personnes, des organisations ou tout être vivant, qui permettent d'éviter ou bien de profiter des effets directs et indirects du changement climatique (Stéphane, 2011). Les méthodes d'adaptation communes dans l'agriculture comprennent la diversification des cultures, la gestion des terres et des eaux, la modification des dates de plantation, changement des plans d'assolement, la diversification des variétés végétales, l'assurance des récoltes, la diversification des revenus, la migration, l'utilisation conjuguée des eaux de surface et souterraine, développement de nouvelles sources d'eau (approfondissement des forage, achat et vente d'eau sur des marchés d'eau informels), vente des terres et reconversion vers l'agriculture pluviale (Smit et Skinner, 2002 ; Moench, 2007 ; Bryan et al., 2009; Molle et al., 2010; Mwinjaka et al., 2010; Jones et Boyd, 2011; Gebrehiwot et van der Veen, 2013; Wheeler et al., 2013; van Steenbergen et al., 2014 ; Alam, 2015; Bosco et al., 2016).

En zones arides la rareté des ressources en eau souterraine aggravée par les risques des changements climatiques impacte à des degrés variables tous les systèmes de production agricole (Rosenzweig et al., 2004 ; Iglesias et Garrote, 2015 ; Lasram et al., 2015) et soulève la question d'adaptation (Faysse et al., 2011 ; Jeder et al., 2013 ; Grami et Ben Rejeb, 2015). L'adaptation à la rareté des ressources naturelles est essentielle, en particulier à la lumière du changement climatique, et peut être poursuivie sous la forme d'une adaptation privée ou publique (Frankhauser et al., 1999; Mendelsohn, 2000; Stern, 2006). La perception et l'adoption des stratégies d'adaptation étaient les deux composantes clés de l'adaptation en agriculture (Maddison,
2007) in Alam (2015) et Elum et al. (2017). Elles sont généralement liées. Selon Yegbemey et al. (2014), l'adaptation des producteurs, face à des stimuli tels que le changement climatique, n'est cohérent qu'au regard de leur conception et donc, de leur perception. Toutes perceptions faussées peuvent conduire à des comportements inappropriés en termes d'adaptation. Cela signifie que les agriculteurs doivent d'abord percevoir un changement dans les conditions climatiques et ensuite mettre en œuvre un ensemble de stratégies pour y remédier.

En agriculture, différentes formes de stratégies d'adaptation peuvent être classées, y compris anticipées (prévues), réactives (autonomes), gestion de la demande et de l'offre, structurelles et non structurelles, et rigides et douces (De perthuis et al., 2010 ; Stéphane, 2011 ; Alam, 2015). L'adaptation peut avoir lieu à la ferme et hors ferme. L'adaptation prévue nécessite une bonne prévision et, souvent, des incitations gouvernementales, alors que l'adaptation autonome se fait par des agents privés (Seo, 2011 ; Alam, 2015). L'adaptation réactive consiste à réagir ex post aux impacts adverses du changement climatique, lorsqu'ils se produisent. L'adaptation anticipative, au contraire, consiste à agir avant que les impacts ne se produisent pour réduire la vulnérabilité à ces impacts et en limiter les conséquences adverses ou en tirer des bénéfices nouveaux (De perthuis et al., 2010 ; Magnan, 2014). Néanmoins, la distinction entre ces deux formes reste non perceptible dans la réalité. En effet, en choisissant de s'adapter aux risques climatiques, le producteur ou l'irrigant adopte des stratégies aussi bien réactives qu'anticipatives. Dans ce processus de double réaction (perception et adaptation), le producteur ne perd pas sa nature d'agent économique. L'étude des pratiques agricoles et des objectifs des agriculteurs peut aider à comprendre ces stratégies dans le sud-est tunisien pris comme zone d'étude.

Dans le sud-est Tunisien, au fil des années, les sociétés locales ont développé la capacité de s'adapter aux changements environnementaux et à la variabilité du climat. L'agriculture irriguée en particulier est désormais fragilisée dans de nombreuses régions. Pourtant, cette catégorie d'exploitations est réputée résiliente : depuis toujours, 
elle a su composer avec les risques climatiques et économiques. En revanche, l'accroissement très prononcé de la variabilité spatiale et temporelle du climat, la faiblesse du dispositif institutionnel d'adaptation à la rareté des ressources en eaux souterraines et de l'ignorance de différentes stratégies d'adaptation privés au défi des changements climatiques posent aujourd'hui des questions cruciales en termes d'adaptabilité des systèmes de production irrigués et constituent des défis majeurs beaucoup plus difficiles à gérer (Jeder et al., 2013). L'impact de ces évènements sur l'agriculture irriguée est en effet multiforme (Frija et al., 2016 ; Mahdhi et al., 2016). Il pèse sur les ressources en eau souterraine (épuisement et salinité), sur les personnes, sur l'augmentation des besoins d'irrigation, sur le capital des exploitations et sur les résultats de ces dernières (systèmes d'élevage et de culture moins productifs), mais également sur les rapports sociaux au sein des sociétés, qui sont mis à mal, le tout contribuant à accroître la vulnérabilité en particulier des petites exploitations familiales, qui ont des capacités limités pour s'adapter. A l'échelle des périmètres irrigués privés, les irrigants se trouvent obligés à investir toujours pour continuer de disposer d'eau douce en quantité suffisante pour leurs cultures soit à modifier leurs systèmes de cultures aux niveaux des périmètres irrigués privés pour s'adapter à la pénurie des ressources en eau souterraine et éviter les effets de la variabilité du climat (Faysse et al., 2011 ; Frija et al., 2014 ). Face à ces évolutions, s'adapter aux effets du changement climatique sur les ressources en eau nécessitera à coté des ajustements ${ }^{11}$ techniques, politiques, institutionnels et comportementaux une bonne compréhension et analyse de différentes stratégies d'adaptation privés à la rareté des ressources en eau souterraine dans le sud-est Tunisienne, pris come zone d'étude. A cette fin, l'objectif de cet étude est d'étudier les différentes types de stratégies adoptées par les agriculteurs pour faire face à une crise d'accès à l'eau souterraine et d'analyser la rentabilité économique des systèmes de production irrigués en relation avec les adaptations aux changements climatiques dans le sud-est Tunisienne, pris come zone d'étude. Le reste de ce travail est structuré de la manière suivante : la Section 2 présente les aspects méthodologiques. la Section 2 présente les aspects méthodologiques. Celle-ci comprend une présentation de la zone d'étude, la collecte des données utilisées et le modèle empirique et conceptuel. La Section 3 présente l'analyse descriptive des données et les résultats de l'estimation du modèle. La conclusion et les enseignements sont présentés dans la Section 4.

\section{Matériel et méthodes}

\subsection{Zone d'étude et sources de données}

La zone d'étude envisagée est la partie nordouest de la Jeffara tunisienne. Elle s'étend sur les deux gouvernorats de Médenine et de Tataouine. Elle s'inscrit dans un quadrilatère passant par Rmada, Ghomrassen, Médenine et Jorf. La superficie de cette zone est d'environ 117000 ha. Sur le plan climatique, cette zone se trouve dans l'aire isoclimatique méditerranéenne (à pluie hivernale et été sec). Son climat, aride à saharien, se caractérise par une pluviométrie faible (inférieure à $200 \mathrm{~mm}$ ) irrégulière et sporadique (le coefficient de variation dépasse $50 \%$ ) et par un bilan climatique déficitaire pour tous les mois de l'année (Genin et al., 2006). Les ressources en eau souterraine représentent le principal apport hydrique exploitable dans cette zone. Ces ressources subissent des fortes pressions qui ne cessent de s'accroître à la suite d'un accroissement démographique rapide et d'une extension remarquable du potentiel irrigable qui atteint 8241 ha en 2015 dont 5925 ha sont des périmètres irrigués privés (PIP), crées principalement autour des puits de surface et des forages (ODS, 2016).

L'agriculture irriguée constitue l'activité économique principale des régions de Smar, Bedoui, Amra, Magrawia, El Maouna et d'El Ferch (Figure 1). Elle est caractérisée par des exploitations de petite taille orientées essentiellement

\footnotetext{
${ }^{1}$ Ces ajustements feront appel à un large éventail de mesures d'ordre structurel (construction de nouveaux ouvrages, réutilisation des eaux usées, entretien des réseaux urbains...) et non-structurel (planification territoriale, politiques fiscales et économiques pour une meilleure gestion de la demande, systèmes d'assurances contre les risques naturels.
} 
Figure 1 - Localisation de la zone d'étude.

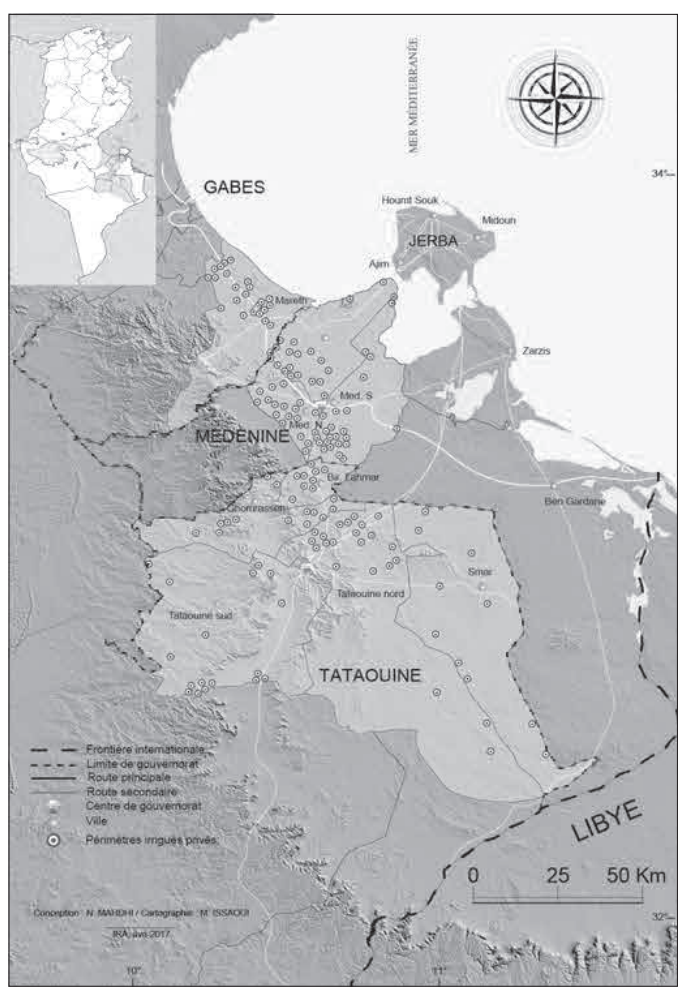

vers les cultures maraîchères de plein champ et de l'arboriculture avec prédominance de l'olivier (Khatoui, 2014 ; Mahdhi, 2016).

Une combinaison d'approches qualitatives (des interviews semi structurées de groupes informels et individuels, des observations participantes et des transects participatifs) et quantitatives, moyennant des enquêtes approfondies, ont été utilisées. Sept périmètres irrigués privés rela- vant aux deux Gouvernorats de Médenine (Zone 1) et de Tataouine (Zone 2) ont été retenus pour l'étude approfondie. La collecte de données a été faite grâce à un questionnaire portant sur 190 exploitants sélectionnés d'une manière aléatoire et proportionnel à l'effectif total des puits de surface par périmètres irrigués. Le Tableau 1 montre la structure de l'échantillon par périmètre.

Les principales données collectées auprès des exploitants échantillonnés étaient relatives à leurs caractéristiques démographiques et socioéconomiques (sexe, âge, exercice d'activité secondaire, niveau d'instruction, expérience en agriculture, contact avec la vulgarisation, nombre d'actifs agricoles par ménage, et droit de propriété sur la terre), aux stratégies développées par eux pour faire face aux conséquences des changements climatiques et aux prix et les quantités des inputs et outputs entrant dans la production de la campagne agricole 2014-2015. Le traitement et l'analyse statistique des données collectées ont été réalisés avec les logiciels Word, Excel et SPSS.

\subsection{Modélisation empirique de la rentabilité : spécification du modèle et variables utilisées}

Dans un contexte de pénurie des ressources en eau souterraine aggravé par les risques de changement climatique dans le sud-est Tunisien, l'irrigant adopte des stratégies aussi bien réactives qu'anticipatives. Dans un premier temps, le producteur réagit au risque auquel il est confronté et dans un deuxième temps anticipe sur celui-ci. Dans ce processus de double réaction, l'irrigant ne perd pas sa nature d'agent économique. Ceci le

Tableau 1 - Structure de l'échantillon d'étude.

\begin{tabular}{|l|l|l|c|c|c|}
\hline $\begin{array}{l}\text { Zone } \\
\text { géographique }\end{array}$ & \multicolumn{1}{|c|}{ Nappe phréatique } & $\begin{array}{l}\text { Périmètres } \\
\text { irrigués }\end{array}$ & $\begin{array}{c}\text { Nombre des puits } \\
\text { de surface }\end{array}$ & $\begin{array}{c}\text { Taille de } \\
\text { l'échantillon }\end{array}$ & $\begin{array}{c}\text { Taux de } \\
\text { sondage (\%) }\end{array}$ \\
\hline \multirow{2}{*}{$\begin{array}{l}\text { Gouvernorat } \\
\text { de Médenine }\end{array}$} & Presqu'île de Jorf & $\begin{array}{l}\text { Magraouia } \\
\text { Bedoui } \\
\text { Amra }\end{array}$ & 271 & 24 & 13 \\
\cline { 2 - 6 } & BV Smar (Médenine) & Smar & 760 & 69 & 36 \\
\hline \multirow{2}{*}{$\begin{array}{l}\text { Gouvernorat } \\
\text { de Tataouine }\end{array}$} & Om El Khialet & El Achouch & 172 & 16 & 8 \\
\cline { 2 - 6 } & Oued Tlalet - El Ferch & El Ferch & 382 & 34 & 18 \\
\cline { 2 - 6 } & Plaine Smar & El Maouna & 519 & 47 & 25 \\
\hline Total & - & - & 2104 & 190 & 100 \\
\hline
\end{tabular}

ODS, 2016 ; CRDA Médenine, 2015, CRDA de Tataouine, 2015. 
ramène, selon le modèle néoclassique, à un comportement rationnel. En effet, dans le processus d'adaptation les agriculteurs sont supposés maximiser leurs revenus nets en utilisant les facteurs de production $(\mathrm{X})$ en fonction des caractéristiques de leurs exploitations et en faisant face aux conditions climatiques $(\mathrm{F})$, et aux prix des facteurs $(\mathrm{R})$. Atteindre le revenu net le plus élevé est fréquemment identifié comme le premier objectif de la plupart des agriculteurs. Pour atteindre ses objectifs, les irrigants doivent choisir de nouvelles combinaisons des facteurs de production agricoles (capital, travail) de tel sorte que les revenus marginaux sont égaux aux coûts marginaux pour toutes les alternatives entreprises. Les conditions écologiques nouvelles (changements climatiques) et la disponibilité en ressources ne sont pas les seuls éléments pris en compte lorsque l'irrigant choisit de réajuster son système de production agricole. Les considérations relatives à l'environnement économique et social pèsent aussi d'un grand poids dans sa décision.

Afin de caractériser le problème auquel est soumis l'irrigant, la forme fonctionnelle du modèle de la rentabilité économique sous la forme COBB-DOUGLAS est retenue dans le cadre de ce travail est la suivante :

$\pi_{i}=e^{\alpha_{0}} \operatorname{Sup}_{i}^{k 1} L_{I}^{K 2} K_{i}^{k 3}\left(\prod_{k=1}^{m} A D P A T_{k i}^{\beta i}\right)\left(\prod_{p=2}^{n} Z_{p i}^{\lambda p}\right) e^{\varepsilon i}$
En appliquant la fonction logarithme népérien à chaque membre de l'équation (1), on obtient :

$$
\begin{gathered}
\ln \left(\pi_{i}\right)=\alpha_{0}+k_{1} \ln \left(\text { Sup }_{i}\right)+k_{2} \ln \left(L_{i}\right)+k_{3} \ln \left(K_{i}\right) \\
+\sum_{k=1}^{3} \beta_{k} A D A P T_{k i}+\sum_{p=1}^{12} \lambda_{p} Z_{p i}+\varepsilon_{i}
\end{gathered}
$$

Le $\pi_{i}$ est le profit de l'irrigant i en DT. Il calculé en considérant deux principales types de cultures à savoir maraicher et arboricole. Pour une culture donnée le profit annuel $\mathrm{RN}_{\mathrm{j}}$ mathématiquement est exprimé par la formule suivante :

$$
R N_{J}=P B V_{J}-C V_{j}
$$

Ici, $\mathrm{PBV}_{\mathrm{j}}$ est le produit brut en valeur ou recette totale, donné par le produit brut physique multiplié par le prix unitaire $\mathrm{p}$ de vente du produit, $\mathrm{CV}_{\mathrm{j}}$ l'ensemble des charges variables imputables à l'activité $\mathrm{j}$ considérée. En prenant en compte toutes les activités de production, le profit pi de l'irrigant i est donné par :

$$
\pi_{i}=\sum_{j=1}^{2} R N_{i j} \text { avec } \mathrm{j}=1,2 ;
$$

où, RNij est le profit annuel de la culture $\mathrm{j} d u$ producteur $\mathrm{i}$.

Les variables $\operatorname{Sup}_{\mathrm{i}}, \mathrm{L}_{\mathrm{i}}$ et $\mathrm{K}_{\mathrm{i}}$ représentent respectivement pour l'irrigant i la superficie irriguée en ha, la main-d'œuvre totale utilisée en nombre de jour et le capital utilisé en DT (intrants agricoles, main-d'œuvre salariée, matériels agricoles, etc.).

Tableau 2 - Description des différentes variables du modèle.

\begin{tabular}{|l|c|c|c|c|}
\hline Noms des variables & Types $^{a}$ & Code & Modalités & Signes attendus \\
\hline Superficie irriguée & C & SUP & - & + \\
\hline Main-d'œuvre totale utilisée & C & L & - & $+/$ - \\
\hline Quantité de capital utilisé & C & CAP & - & + \\
\hline Age & D & AGE & - & + \\
\hline Niveau d'instruction & D & N. INS & $\begin{array}{c}1=\text { secondaire et plus } \\
\text { et } 0=\text { autres }\end{array}$ & + \\
\hline Situation matrimoniale & D & SITMATRI & $\begin{array}{c}0=\text { Non marié } ; \\
1=\text { Marié }\end{array}$ & $+/$ - \\
\hline Activité secondaire & D & ACTSEC & $0=$ Non; $1=$ Oui & $+/$ - \\
\hline Contact avec un vulgarisateur & D & CONTVUL & $0=$ Non; $1=$ Oui & $+/-$ \\
\hline Stratégie offensif & D & Soff & $0=$ Non; $1=$ Oui & + \\
\hline Stratégie défensif & D & Sdéf & $0=$ Non; $1=$ Oui & + \\
\hline Stratégie contractive & D & Scontr & $0=$ Non; $1=$ Oui & + \\
\hline
\end{tabular}

${ }^{a}$ Types $: D=$ variables discontinues $; C=$ variables continues . 
Tableau 3 - Caractéristiques socio-économiques et démographiques des exploitations enquêtées.

\begin{tabular}{|l|c|c|}
\hline Variables qualitatives & Valeurs absolues & Fréquences relatives (\%) \\
\hline Situation matrimoniale & 128 & 85,906 \\
\hline Droit de propriété sur la terre & 128 & 86 \\
\hline Activité secondaire & 47 & 31,757 \\
\hline Contact avec un vulgarisateur & 52 & 35,135 \\
\hline Accès au crédit & 25 & 16,892 \\
\hline Variables quantitatives & Moyennes & Écarts types \\
\hline Age & 50 & 15,726 \\
\hline Expérience & 22,601 & 14.652 \\
\hline Nombre d'actifs agricole & 4,905 & 2,467 \\
\hline Quantité totale de main d'œuvre utilisé & 394,4 & 238,99 \\
\hline Superficie irriguée & 2,627 & 2,079 \\
\hline
\end{tabular}

Les ADAPTi sont les variables liées aux stratégies d'adaptation identifiées. Les $\mathrm{Zi}$ sont les variables relatives aux caractéristiques socio-économiques et démographiques de l'irrigant $i$. Toutes les variables mises dans le modèle sont décrites dans le Tableau 2. Les coefficients $\beta$, $\mathrm{k}$ et $\lambda$ sont les paramètres à estimer et les $\varepsilon_{\mathrm{i}}$ sont les termes d'erreur. En particulier les k représentent les élasticités du profit par rapport aux facteurs de production et les $\beta$ permettent de mesurer les impacts des différentes stratégies sur le profit obtenu.

Le modèle a été estimé par la méthode des Moindre Carrés Ordinaires (MCO). De plus, des tests de Durbin-Waston, de Goldfeld et Quandt et de Farrar-Glauber ont été réalisés pour vérifier respectivement si des erreurs d'auto-corrélation, d'hétéro-cédasticité et de multi-colinéarité n'existent pas significativement. En particulier, les erreurs de multi-colinéarité ont été corrigées par la méthode d'estimation par étapes qui éliminent au fur et à mesure les variables fortement corrélées à d'autres dans le modèle jusqu'à l'obtention d'une estimation sans erreur de multi-colinéarité.

\section{Résultats et discussion}

\subsection{Caractéristiques socio-économiques et démographiques des exploitants}

Les caractéristiques démographiques et socio-économiques des producteurs interrogés sont résumées dans le tableau 3. L'âge moyen de chef d'exploitation familiale est de l'ordre de 50 ans, dont le plus jeune est à l'âge de 17 ans et celui le plus âgé est de l'ordre de 80 ans. La répartition des chefs d'exploitation par sexe est sans objet, ils sont tous des hommes pour l'ensemble des observations sauf une seule exploitation. Cette situation est normale en ce sens que la femme n'accède à ce statut que s'il n'y a pas dans la maison, un homme, en âge d'occuper cette fonction.

La situation matrimoniale fait ressortir $86 \%$ de mariés et $14 \%$ de célibataires. Ces derniers occupent cette fonction de chef d'exploitation du fait de l'absence de père. Le niveau d'instruction reste relativement faible avec $26,3 \%$ de la population ayant atteint le niveau secondaire et 13,2\% seulement de l'échantillon ayant fait des études supérieures, l'un tiers s'arrêtant au primaire. Le pourcentage des individus n'ayant reçu aucune instruction dans les exploitations enquêtées est de l'ordre de $28,4 \%$. Les modes de faire-valoir de la terre dans la zone d'étude sont l'héritage, le don, l'achat, et la location. L'accès à la terre à travers ces modes confère différents droits de propriété. Ainsi, $86 \%$ des personnes interrogées ont affirmé être propriétaires de leurs terres.

L'agriculture est l'activité principale de $68 \%$ des enquêtés. En plus de l'agriculture, $49 \%$ des producteurs interrogés possèdent une activité secondaire. Le commerce et les petits métiers sont les activités secondaires les plus exercées. 
Le nombre d'actifs agricoles par ménage est en moyenne de 4,905 $( \pm 2,467)$ personnes et l'expérience en agriculture est de 22,601 $( \pm 14,65)$ ans. Par ailleurs, il ressort également du tableau que les enquêtés emblavent en moyenne 2,627 ha $( \pm 2,07 \%)$ et consacrent à l'agriculture une moyenne de 394,4 HJ par an. Enfin, les exploitants ont un faible accès au crédit. L'analyse des sources de financement des équipements montre que $17 \%$ des agriculteurs ont bénéficié de subventions, $28 \%$ du matériel a été financé par le recours au crédit agricole et $72 \%$ par les moyens propres de l'exploitation et le recours au crédit informel.

\subsection{Perception du changement climatique}

Partant de l'hypothèse que la capacité d'un irrigant à s'adapter à la pénurie des ressources en eau souterraine dépend de sa perception du phénomène, cette partie analyse les perceptions du changement climatique à partir des réponses des irrigants aux questions suivantes : Sur les 20 dernières années, avez-vous perçu un (des) changement (s) d'un ou de plusieurs facteurs climatiques au niveau de votre exploitation agricole ? Si oui, quel (s) changement (s) avez-vous observé concernant ce (s) facteur (s) ? en quoi consistent ces changements ? "Sur la base des différentes réponses, nous avons constitué les types de changements climatiques.

La quasi-totalité des irrigants interrogés dans la zone d'étude (95\%) a perçu des changements de facteurs climatiques durant les vingt dernières années qui ont des répercussions directes sur le secteur irriguée. Les principales manifestations de ses changement sont expliquées par une baisse des précipitations, un dérèglement de la saison des pluies, une plus grande irrégularité des pluies, une augmentation des températures et des vents plus violents et par l'augmentation du taux d'occurrence des phénomènes météorologiques extrêmes (Figure 2).

L'analyse de la Figure 2 montre que les irrigants perçoivent clairement les changements climatiques. 90,4\% des irrigants perçoivent que les précipitations ont changé. La diminution des pluies $(82,7 \%$ des irrigants interrogées) et des pluies de plus en plus aléatoires et sporadiques (75\% des irrigants interrogées) sont les principales perceptions des changements liés aux précipitations. Le dérèglement de la saison des pluies est perçu par $76,6 \%$ des exploitants. Ce phénomène se manifeste par une diminution de la durée de la saison des pluies, la réduction du nombre de jours de pluie $(81,7 \%)$ et la réduction de l'intensité des précipitations (53,3\%). Cela engendre une pénurie des ressources en eau souterraine (de point de vue quantité et qualité) qui rend plus perceptible la baisse des précipitations. Viennent ensuite le décalage des saisons avec une tendance à la réduction de la saison d'hiver (56,3\% des irrigants interrogées). Quant à la température, $87,3 \%$ des enquêtés perçoivent des variations thermiques se manifestant par l'augmentation du nombre de jours chauds $(70,1 \%)$, l'augmentation des vagues de chaleur $(84,3 \%$ des irrigants interrogées) et le changement des extrêmes de températures $(87,1 \%$ des irrigants

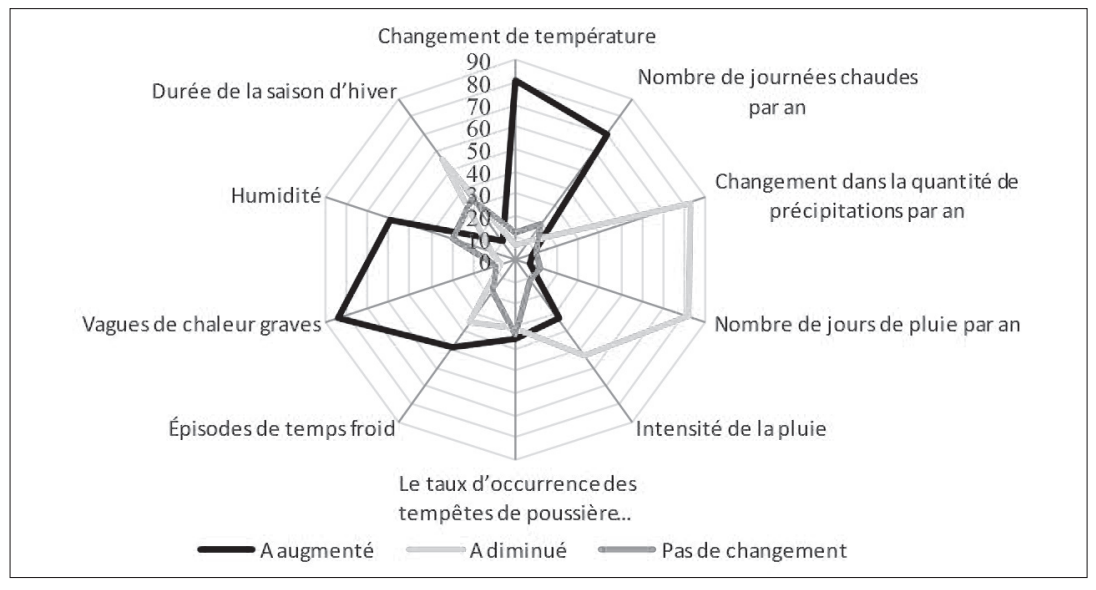

Figure 2 - Taux de perception des changements de facteurs climatiques en $\%$. 
interrogées) qui se traduit par des périodes plus chaudes ou plus froides. Enfin, $68,5 \%$ des producteurs interrogées percevaient d'autres changements tels que des vents plus forts (36\%) et une augmentation de l'humidité $(59,4 \%)$. Ces résultats concordent avec les études mentionnées dans la littérature qui s'accordent sur une augmentation de la fréquence et de l'intensité des années extrêmes sèches et une baisse de la pluviométrie moyenne qui se traduirait par une réduction des ressources en eau disponibles (MEDD/DGEQV, 2008; MAE et GIZ, 2011 ; Lasram et al., 2015). Les résultats obtenus ont par ailleurs corroboré ceux de Maddison (2007), Mertz et al. (2009), Paraïso et al. (2012), Omar et al. (2015) et Lasram et al. (2015) qui révèlent que les populations se rendent compte de la variabilité du climat.

\subsection{Stratégies d'adaptation diversifiées face à la pénurie d'eau}

Cette section analyse les stratégies d'adaptations des irrigants à la pénurie des ressources en eau souterraine dans un contexte de changement climatique à travers la réponse à la question suivante : « quels ajustements avez-vous faits dans votre système de production agricole afin de vous adapter aux changements que vous avez observés ? ».
Les stratégies d'adaptation aux changements climatiques sont diverses dans la zone d'étude. Quatre-vingt-cinq pour cent $(85 \%)$ des irrigants enquêtés ayant perçu des changements climatiques et les problèmes de la rareté des ressources en eau souterraine, ont développé une gamme très variée de mesures d'adaptation (Figure 3). Les irrigants ne développant aucune stratégie d'adaptation ont mentionné, entre autres, le manque d'information sur les stratégies d'adaptation et l'absence de réserves financières comme principales barrières à l'adaptation. Ces résultats ont corroboré les observations faites par d'autres études en Afrique (Adger et al., 2005 ; Reidsma et al., 2010 ; Gebrehiwot et van der Veen, 2013; Alam, 2015; Yegbemey et al., 2014) qui révèlent que l'adaptation peut être limitée par de nombreux facteurs, tels que les obstacles socioéconomiques, institutionnels, biophysiques, psychologiques et financiers, et, par conséquent, l'adaptation privée ne peut pas être entièrement autonome.

En termes de stratégies d'adaptation, la Figure 3 illustre trois groupes de stratégie autonome et réactive. Une première catégorie dite d'offensif ou « de chasse d'eau ». Elle est conçue pour la gestion de l'offre en eau d'irrigation et accroitre la production. Elle comprend, principalement,

Figure 3 - Mesures et stratégies d'adaptation répertoriées.

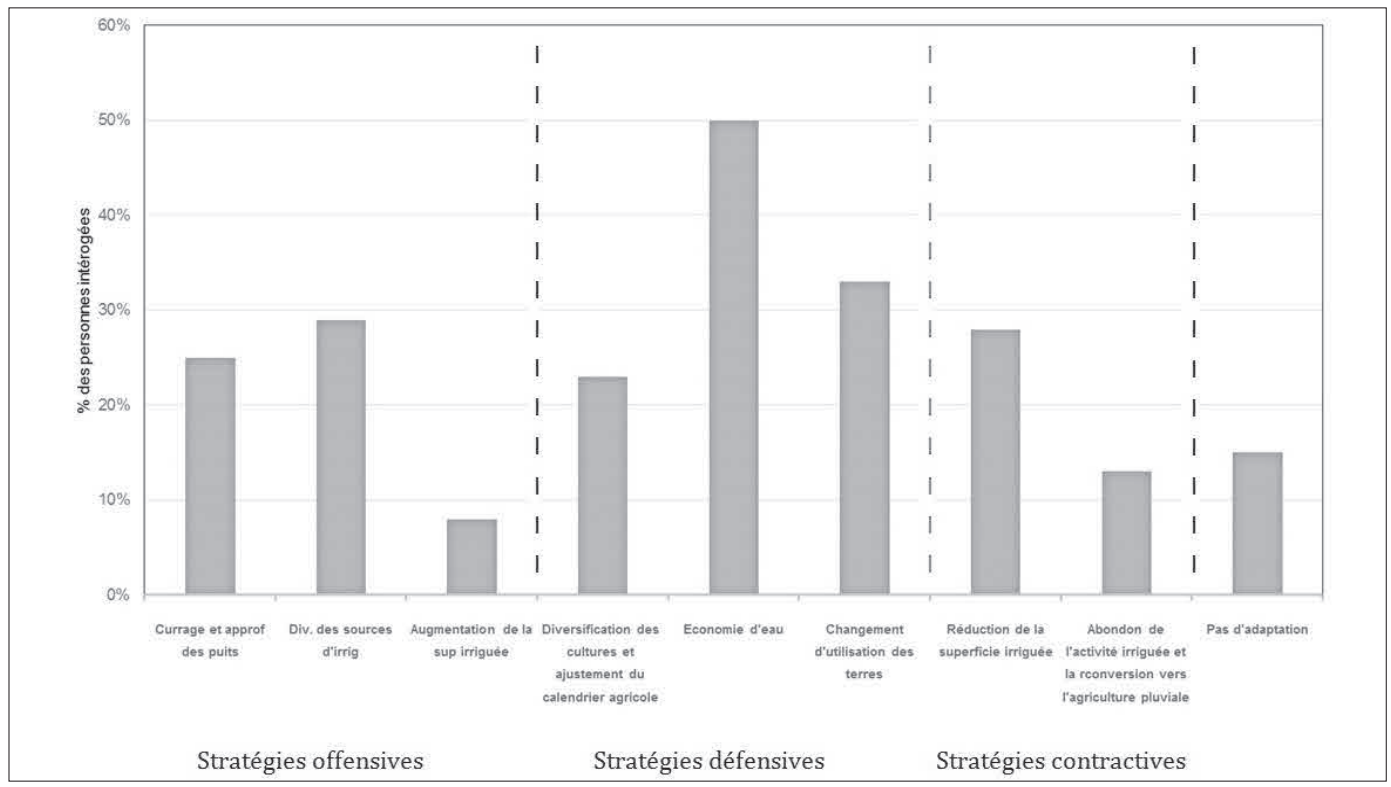


l'approfondissement et le curage des nouveaux puits, la diversification des sources d'irrigation (branchement au réseau public d'irrigation) et l'augmentation de la superficie irriguée à travers la location des terres plus riche en eau. Elle est adoptée par $62 \%$ des irrigants. En moyenne ses actions sont considérées comme des mesures d'adaptations positives dans la mesure où elle améliore la capacité des irrigants à une telle variabilité climatique mais les problèmes de durabilité dans la conception et la mise en œuvre de ces mesures d'adaptation restent posés dans une zone marquée par la surexploitation des ressources en eau souterraine. La deuxième catégorie est dite défensive ou d'adaptatif. Elle est conçue pour la gestion de la demande en eau d'irrigation à l'échelle de l'exploitation. Elle vise à adapter les systèmes de production à l'eau disponible sur l'exploitation compte-tenu des puits et forages existants. Elle comprend la diversification des cultures, les ajustements du calendrier agricole, le changement du système de culture et conservation de l'eau d'irrigation (économie de l'eau) à l'échelle de l'exploitation. Elle est adoptée par $83 \%$ des irrigants. En moyenne ses actions sont considérées comme des mesures d'adaptation positives et générales que les exploitations Tunisienne devront continuer d'adopter à l'avenir pour améliorer la capacité d'adaptation des irrigants à une telle variabilité climatique et maintenir la productivité du secteur irrigué à travers une amélioration de l'infrastructure de l'irrigation et de pratiques de gestion ainsi que le passage à des cultures qui valorise mieux l'eau d'irrigation. Enfin, la troisième catégorie est dite contractive consistant à diminuer l'échelle de fonctionnement de l'exploitation par le recours à la diminution de la superficie irriguée et la reconversion vers l'agriculture pluviale. Elle est adoptée par $41 \%$ des irrigants. Ce type de stratégie est considéré comme négatif et met en péril la productivité et la durabilité des types d'exploitations qui entretiennent ses types d'actions à l'avenir.

$\mathrm{Eu}$ égard à la littérature, ces résultats ont corroboré les résultats d'autres études en Afrique du Nord (Berahmani et al., 2012; Grami et Ben Rejeb, 2015 ; Frija et al., 2016), en Asie et du Moyen-Orient (Molle et al., 2010; Venot et al.,
2010; Ghazouani et al., 2014 ; Alam, 2015), en Europe (Iglesias et Garroteb, 2015) et en Australie (Wheeler et al., 2013).

Ces actions et stratégies individuelles montrent à la fois des points communs et des spécificités par rapport à d'autres stratégies d'adaptation avancées par d'autres travaux. Ces actions ont été identifiées au niveau des périmètres irrigués où les agriculteurs sont confrontés à une pénurie d'eau de surface (Molle et al., 2009). En Asie du Sud dans de nombreuses situations d'accès difficile aux ressources en eau souterraine, ces actions comprennent l'achat et vente d'eau sur des marchés d'eau informels (Mukherji et Shah, 2005). Dans quelques cas, ces stratégies d'adaptation peuvent être collectives comme le cas de la région de Souss, au Maroc où les agriculteurs ont collectivement converti leur système d'irrigation en irrigation localisée face à la baisse des débits des forages collectifs, et à la région de Nadhour (Nord de la Tunisie) ou le GDA « Groupement de Développement Agricole » arrêtent de donner de l'eau à des exploitations agricoles situées hors du périmètre et limitent au début de l'année les superficies irriguées par agriculteur pour diminuer les tensions sur le tour d'eau (Faysse et al., 2011). Néanmoins, la plupart de ses stratégies offensives ou défensives ne sont pas économiquement et écologiquement viables en même temps et leur durabilité reste cependant conditionnée par la qualité des intervenants du côté des usagers comme de l'administration.

\subsection{Rentabilité économique des stratégies d'adaptation}

Dans un contexte de raréfaction des ressources en eau souterraine aggravé par les risques de changements climatiques, l'adaptation apparaît comme une nécessité pour les irrigants dans le sud-est Tunisien. Cependant, il ne faudrait pas perdre de vue que l'effet ou le résultat attendu par les irrigants en adoptant la plus simple ou la plus complexe des possibles stratégies d'adaptation reste le maintien de son niveau de profit. Dans le meilleur des cas, une amélioration voire augmentation du dit niveau est le résultat escompté. Le Tableau 4 présente quelques éléments du compte d'exploitation des enquêtés. 
Tableau 4 - Quelques éléments du compte d'exploitation de l'irrigant selon le type d'adaptation ( $\mathrm{N}=190)$.

\begin{tabular}{|l|c|c|c|c|c|}
\hline \multicolumn{2}{|c|}{ Stratégies d'adaptation } & $\begin{array}{c}\text { Produit brut } \\
(\text { DT/ha })\end{array}$ & $\begin{array}{c}\text { Coûts totaux de } \\
\text { production }(D T / h a)\end{array}$ & $\begin{array}{c}\text { Marge nette } \\
(D T / h a)\end{array}$ & $\begin{array}{c}\text { Profit annuel } \\
(D T)\end{array}$ \\
\hline \multirow{4}{*}{ Offensif } & Oui & $\begin{array}{c}11139 \\
( \pm 11503,23)\end{array}$ & $\begin{array}{c}2427 \\
( \pm 1742,211)\end{array}$ & $\begin{array}{c}7062 \\
( \pm 6209,401)\end{array}$ & $\begin{array}{c}37359 \\
( \pm 46638)\end{array}$ \\
\cline { 2 - 6 } & Non & $\begin{array}{c}2907 \\
( \pm 1736,340)\end{array}$ & $\begin{array}{c}1744 \\
( \pm 681,894)\end{array}$ & $\begin{array}{c}3314 \\
( \pm 2538,832)\end{array}$ & $\begin{array}{c}8171 \\
( \pm 8105,2597)\end{array}$ \\
\hline \multirow{5}{*}{ Défensif } & Oui & $\begin{array}{c}6443 \\
( \pm 7025)\end{array}$ & $\begin{array}{c}1447 \\
( \pm 777,96)\end{array}$ & $\begin{array}{c}5047 \\
( \pm 5433,639)\end{array}$ & $\begin{array}{c}22069 \\
( \pm 27466,780)\end{array}$ \\
\cline { 2 - 6 } & Non & $\begin{array}{c}2907 \\
( \pm 1736,340)\end{array}$ & $\begin{array}{c}1744 \\
( \pm 681,894)\end{array}$ & $\begin{array}{c}3314 \\
( \pm 2538,832)\end{array}$ & $\begin{array}{c}8171 \\
( \pm 8105,2597)\end{array}$ \\
\hline \multirow{4}{*}{ Contractive } & Oui & $\begin{array}{c}2989 \\
( \pm 1509,964)\end{array}$ & $\begin{array}{c}1360 \\
( \pm 522,436)\end{array}$ & $\begin{array}{c}4443 \\
( \pm 3038,342)\end{array}$ & $\begin{array}{c}10037 \\
( \pm 6735,927)\end{array}$ \\
\cline { 2 - 6 } & Non & $\begin{array}{c}2907 \\
( \pm 1736,340)\end{array}$ & $\begin{array}{c}1744 \\
( \pm 681,894)\end{array}$ & $\begin{array}{c}3314 \\
( \pm 2538,832)\end{array}$ & $\begin{array}{c}8171 \\
( \pm 8105,2597)\end{array}$ \\
\hline \multirow{4}{*}{ Total } & Oui & $\begin{array}{c}6857 \\
( \pm 6754,856)\end{array}$ & $\begin{array}{c}1744,667 \\
( \pm 1067,355)\end{array}$ & $\begin{array}{c}5517,333 \\
( \pm 4727,290)\end{array}$ & $\begin{array}{c}231555 \\
( \pm 27403,346)\end{array}$ \\
\cline { 2 - 6 } & Non & $\begin{array}{c}2907 \\
( \pm 1736,340)\end{array}$ & $\begin{array}{c}1744 \\
( \pm 681,894)\end{array}$ & $\begin{array}{c}3314 \\
( \pm 2538,832)\end{array}$ & $\begin{array}{c}8171 \\
( \pm 8105,2597)\end{array}$ \\
\hline
\end{tabular}

Les chiffres entre parenthèse représentent les écart-types.

Source : Résultats d'analyse des données de recoupement des enquêtes, 2014 et 2015.

D'une manière générale, comparativement aux irrigants n'adoptant aucune des stratégies précédemment identifiées, les irrigants adoptants enregistrent à l'hectare un produit brut moyen, une marge nette moyenne et un profit annuel moyen plus élevés. La tendance évolutive des éléments du compte d'exploitations est la même pour les stratégies défensives et contractives. Tous les groupes d'adaptation entrainent une augmentation du produit brut de la marge nette et du profit annuel du producteur. Par ailleurs, tous les types d'adaptation à l'exception de la stratégie offensive entrainent une réduction des coûts totaux de production (Tableau 5).

\subsection{Déterminants du profit net du producteur}

Dans l'optique d'identifier les déterminants du profit net du producteur, un modèle de régression a été estimé. Les résultats de ce modèle sont présentés dans le Tableau 6. La lecture de ce tableau montre que le modèle de régression est significatif à $1 \%$. Ainsi, $53 \%$ des variations du profit annuel des producteurs enquêtés sont expliquées par les variations des variables introduites dans le dit modèle. Les $47 \%$ de variations du profit annuel non expliquées par les variations des variables introduites dans le modèle seraient attribuables aux facteurs difficilement mesurables tels que le niveau de fertilité des sols, les

Tableau 5 - Tendances évolutives de quelques éléments du compte d'exploitation sous les différents types d'adaptation.

\begin{tabular}{|l|c|c|c|c|}
\hline Stratégies d'adaptation & Produit brut & Coûts totaux & Marge nette & Profit annuel \\
\hline Offensive & +++ & +++ & +++ & +++ \\
\hline Défensive & +++ & --- & +++ & +++ \\
\hline Contractive & +++ & --- & +++ & +++ \\
\hline Tendance générale & +++ & +++ & +++ & +++ \\
\hline
\end{tabular}

+++ Augmentation de valeur ; --- Diminution de valeur. 
Tableau 6 - Déterminants du profit net du producteur.

\begin{tabular}{|l|c|c|c|c|}
\hline Variable & Coefficient & Std. Error & $t$-Statistic & Prob. \\
\hline Constante & 7,72 & 1,74 & 1,078 & 0,347 \\
Ln sup & $0,670^{* * *}$ & 0,190 & 3,508 & 0,000 \\
Ln MO & $0,364^{* *}$ & 0,205 & 1,77 & 0,049 \\
Ln CAP & $-0,056^{*}$ & 0,125 & $-0,448$ & 0,075 \\
Age & $-0,008$ & 0,008 & $-0,979$ & 0,330 \\
Niv d'ins & $-0,153$ & 0,222 & $-0,690$ & 0,491 \\
Etat civil & $-0,252$ & 0,216 & $-1,163$ & 0,247 \\
F.agricole & $0,677^{*}$ & 0,394 & 1,716 & 0,089 \\
Mfv & 1,109 & 0,730 & 1,519 & 0,132 \\
STG D & $5,974^{* * *}$ & 1,623 & 3,680 & 0,000 \\
STGOFF & $6,416^{* * *}$ & 1,604 & 3,998 & 0,000 \\
STRG C & 6,305 & 1,579 & 3,992 & 0,115 \\
Vulg & 0,169 & 0,253 & 0,667 & 0,505 \\
Ancienneté & $-0,006^{* *}$ & 0,008 & -0.692 & 0,049 \\
Activ sec & $0,463^{* *}$ & 0,238 & 1,1942 & 0,055 \\
\hline Variable dépendante & \multicolumn{4}{|c|}{ Log népérien du profit annuel Lnprofit } \\
Nombre d'observation & \multicolumn{3}{|c|}{190} \\
Statistique F de Fisher & $73,7 * * *$ (DDL1=14 ; DDL2=134); p=0,0001 \\
R ${ }^{2}$ & \multicolumn{3}{|c|}{0,53} \\
\hline
\end{tabular}

*** : significatif à $1 \%$; ** : significatif à $5 \%$; * : significatif à $10 \%$.

conditions climatiques et les divers changements qu'on peut enregistrer d'une saison à l'autre. Les résultats obtenus indiquent que les variables telles que la superficie irriguée, les quantités totales de main-d'œuvre et de capital utilisées, la formation agricole, l'ancienneté de l'activité, l'activité secondaire et son type d'adaptation (stratégies offensive et défensive) sont les déterminants de son niveau de profit annuel réalisé. $\mathrm{La}$ superficie irriguée, la stratégie offensive et celle défensif ont des effets positifs et significatifs à $1 \%$ sur le profit annuel réalisé par l'irrigant. La quantité totale de main-d'œuvre, l'activité secondaire et l'ancienneté de l'activité ont par contre des effets significatifs positifs pour les deux premiers mais négatifs pour le dernier à $5 \%$. L'âge, la situation matrimoniale, le contact avec l'agent de vulgarisation et l'accès n'ont pas d'effets significatifs sur le profit annuel du producteur.

Le nombre et la diversité des adaptations mises en place par les irrigants indiquent une réelle volonté de minimiser la pénurie des ressources en eau souterraine et les impacts des changements climatiques. Selon la stratégie adoptée par le producteur, des répercutions sont observées sur les éléments de son compte d'exploitation. Les résultats du modèle de régression indiquent que seuls la stratégie offensive et la stratégie adaptative ont des effets significatifs sur le profit annuel du producteur. Ces 2 types d'adaptations apparaissent alors comme les plus économiquement rentables. Dans un contexte où l'irrigant s'adapte aux changements climatiques tout en poursuivant ses objectifs de maximisation de son profit, la stratégie offensive et la stratégie adaptative apparaissent alors comme les stratégies d'adaptation les plus indiquées.

Le profit annuel est aussi significativement influencé par la superficie emblavée, les quantités de main d'ouvre utilisées, le capital utilisé, la formation agricole, l'ancienneté de l'activité et de l'activité secondaire de l'exploitant. L'effet significatif et positif de la superficie est attribuable au fait que les petites superficies appartiennent à des exploitants dont l'objectif principal de production est l'autoconsommation. Ces derniers ne cherchant pas un profit substantiel. Le capital utilisé, bien que positif à des effets négatifs. Ceci va de l'efficacité du producteur dans l'allocation de ces facteurs de production. 


\section{Conclusion}

L'accroissement très prononcé de la variabilité spatiale et temporelle du climat constitue de nos jours des défis majeurs beaucoup plus difficiles à gérer en zone aride et soulève la question d'adaptation. L'objectif assigné à ce papier est d'étudier les stratégies d'adaptations des irrigants à la pénurie des ressources en eau souterraine dans un contexte de changement climatique dans le sud-est Tunisien. Du point de vue méthodologique, l'approche par enquêtes socioéconomiques auprès de 190 exploitations agricoles, et le modèle de la rentabilité économique de COBB-DOUGLAS nous ont permis d'étudier les différentes types de stratégies adoptées par les agriculteurs et d'analyser la rentabilité économique des systèmes de production irrigués en relation avec les adaptations aux changements climatiques. Huit réponses d'adaptation ont été identifiées. Ses mesures comprennent le forage de nouveaux puits et le curage annuel des puits existants, la diversification des sources d'irrigation, l'augmentation de la superficie irriguée, le recours à l'économie de l'eau, le changement des systèmes de cultures, la diversification des cultures et les ajustements du calendrier agricole, la réduction de la superficie irriguée, l'abandon de l'activité irrigué et l'orientation vers l'agriculture pluviale.

En termes de stratégies d'adaptations ces pratiques de gestion sont classées en trois types de stratégies potentiels : une stratégie qualifiée d'adaptative ou de défensive (S1), une stratégie qualifiée d'offensive ou de « chasse » d'eau (S2) et une stratégie qualifiée de contractive (S3). Selon la stratégie adoptée par le producteur, des répercutions sont observées sur les éléments de son compte d'exploitation.

Eu égard à la littérature, ces différentes mesures d'adaptation apparaissent pertinentes. La connaissance de différentes stratégies d'adaptation permet d'identifier des options d'adaptation à la fois réalistes du point de vue de leur mise en œuvre et ambitieuses du point de vue de leurs objectifs et aider à élaborer des priorités pour l'adaptation des ressources en eau pour l'irrigation.

Néanmoins, si certaines sont bien adaptées aux changements décrits (diversification des sources d'irrigation, conservation de l'eau d'ir- rigation), d'autres sont susceptibles d'aggraver les impacts des changements climatiques et d'engendrer des pressions sur les ressources en eau souterraine (généralisation des stratégies offensives) et met en question la durabilité de ses pratiques sur le plan économique et environnementale et accroître le risque pour le secteur irriguée et de la vulnérabilité en particulier des petites exploitations familiales, qui ont des capacités limités pour s'adapter. La Tunisie doit réagir dès maintenant en fonction de la prévision du climat futur. Des voies alternatives doivent donc être trouvées, à la fois en termes de stratégies, mais aussi pour faire participer les différents acteurs à la conception de ces stratégies afin de réduire les impacts du changement climatique sur le secteur irriguée. Dans ce sens, des mesures d'adaptation doivent être prises dans différentes conditions agro-écologiques et climatiques à travers l'encouragement de la recherche en matière d'évaluation et d'identification des options d'adaptation (paquet technologique agricole adapté aux changements climatiques, diffusion de nouvelles variétés tolérantes et de variétés précoces adaptables aux changements climatiques, etc.) à la fois réalistes du point de vue de leur mise en œuvre et ambitieuses du point de vue de leurs objectifs. Dans le court et le moyen terme, les possibilités d'adaptation du pays doivent passer par une accélération de la mobilisation de nouvelles ressources (construction des barrages et la redistribution de l'eau des barrages vers l'agriculture, recyclage et la récupération des eaux usées pour l'agriculture), recharge de la nappe, collecte des eaux de ruissèlement, intensification de l'irrigation, tarification incitative, amélioration de l'efficacité d'usage de l'eau d'irrigation à travers la génération de l'irrigation goutte à goutte et la réduction des pertes en eau. D'autres mesures peuvent être liées aux pratiques culturales telles la refonte progressive des calendriers agricoles traditionnels, l'optimisation des dates de semis en fonction des changements du climat, l'utilisation de semences sélectionnées et le choix de variétés à cycle court et résistantes à la sécheresse, et enfin la reconversion et le repositionnement des cultures selon l'évolution du contexte bioclimatique. Ce choix serait cependant cau- 
tionné par un accompagnement technique et financier des agriculteurs. Enfin des mesures d'accompagnement (formation, encadrement, financement, infrastructure) doivent être prises pour faciliter l'adaptation et aider à élaborer des priorités pour l'adaptation des ressources en eau pour l'irrigation en zone aride.

\section{Bibliographie}

Adger W.N., Arnell N.W., Tompkins E.L., 2005. Successful adaptation to climate change across scales. Global Environmental Change, 15: 77-86.

Alam K., 2015. Farmers' adaptation to water scarcity in drought-prone environments: a case study of Rajshahi district, Bangladesh. Agricultural Water Management, 148: 196-206.

Bekkar Y., Kuper M., Errahj M., Faysse N., Gafsi M., 2009. On the difficulty of managing an invisible resource; farmers' strategies and perceptions of groundwater use, field evidence from morocco. Irrigation and Drainage, 58: 252-263.

Berahmani A., Faysse N., Errahj M., Gafsi M., 2012. Chasing water: diverging farmers' strategies to cope with the groundwater crisis in the coastal Chaouia region in Morocco. Irrigation and Drainage, 61: 673-681.

Bosco J.K., Vodounouy Y.O.D., 2016. Agriculture paysanne et stratégies d'adaptation au changement climatique au Nord-Bénin, Cybergeo. European Journal of Geography, http://journals.openedition. org/cybergeo/27836; DOI:10.4000/cybergeo.27836.

Bryan E., Temesgen T.D., Glwadys A.G, Claudia R., 2009. Adaptation to climate change in Ethiopia and South Africa: options and constraints. Environmental Science \& Policy, 12(4): 413-426.

CRDA (Commissariat de Développement Agricole) de Médenine, 2015. Rapport annuel d'Arrondissement des périmètres irrigués (en arabe), $25 \mathrm{pp}$.

CRDA (Commissariat de Développement Agricole) de Tataouine, 2015. Rapport annuel d'Arrondissement des périmètres irrigués (en arabe), $20 \mathrm{pp}$.

De Perthuis C., Hallegatte S., Lecocq F., 2010. Economie de l'adaptation au changement climatique. Rapport du Conseil Economique pour le Développement Durable, France. Ministère de l'Ecologie, de l'Energie, du Développement Durable et de la Mer. http://www.ladocumentationfrancaise.fr/var/storage/ rapports-publics/104000108.pdf.

Elum Z.A., Modise D.M., Marr A., 2017. Farmer's perception of climate change and responsive strategies in three selected provinces of South Africa. Climate Risk Management, 16: 246-257.
Faysse N., Hartani T., Frija A., Marlet S., Tazekrit I., 2011. Agricultural Use of Groundwater and Management Initiatives in the Maghreb: Challenges and Opportunities for Sustainable Aquifer Exploitation. Economic Brief, African Development Bank, 24 pp. http://www.afdb.org/fileadmin/uploads/afdb/Documents/Publications/Brochure\%20Anglais\%20agriculture $\% 20$ et $\% 20$ eau_North $\% 20$ Africa $\% 20$ Quater$1 \mathrm{\%} \% 20$ Analytical.pdf.

Frija A., Chebil A., Speelman S., 2016. Farmes' adaptation to groundwater shortage in the dry areas: improving appropriation or enhancing accommodation? Irrigation and Drainage, 65(5): 691-700.

Frija A., Chebil A., Speelman S., Faysse N., 2014. A critical assessment of groundwater governance in Tunisia. Water Policy, 16: 358-373.

Gebrehiwot T., van der Veen A., 2013. Adaptation to Climate Change in Agriculture. Environmental Management, 52: 29-44.

Génin D., Guillaume H., Ouessar M., Ouled Belgacem A., Romagny B., Sghaier M. et al., 2006. Entre Désertification et Développement la Jeffara Tunisienne, IRA, IRD, Tunis: Ed. Cérès.

Ghazouani W., Molle F., Swelam A., Rap E., Abdo A., 2014. Understanding farmers' adaptation to water scarcity: a case study from the western Nile Delta, Egypt. Colombo, SriLanka, International Water Management Institute (IWMI), IWMI Research Report, 160, $31 \mathrm{pp}$.

GIEC, 2001. Bilan 2001 des changements climatiques: les éléments scientifiques. Rapport du groupe de travail I du GIEC. Contribution du groupe de travail I au troisième rapport d'évaluation du GIEC, 97 pp. http://www.ipcc.ch/pub/un/giecgt1.pdf.

Grami D., Ben Rejeb J., 2015. L'impact des changements climatiques sur le rendement de la céréaliculture dans la Région du Nord-Ouest de la Tunisie (Béja). New Medit, 4: 36-41.

Iglesias A., Garroteb L., 2015. Adaptation strategies for agricultural water management under climate change in Europe. Agricultural Water Management, 155: 113-124.

Jeder H., Ben Khalifa A., Sghaier M., 2013. Impact des changements climatiques sur l'agriculture dans la plaine de Jeffera au sud-est tunisien. Journal of Agriculture and Environment for International Development, 107(2): 229-242.

Jones L., Boyd E., 2011. Exploring social barriers to adaptation: Insights from Western Nepal. Global Environnemental Change, 21: 1262-1274.

Khatoui Ch., 2014. Stratégies des irrigants confrontés à la rareté des ressources en eau souterraines : Cas des périmètres irrigués privés de Smar (Gouver- 
norat de Tataouine). Projet de fin d'étude, Ecole Supérieure d'Agriculture de Mograne, Tunisie.

Lasram A., Dellagi H., Masmoudi M.M., Ben Mechlia N., 2015. Productivité de l'eau du blé dur irrigué face à la variabilité climatique. New Medit, 1: 61-66.

Magnan A., 2014. De la vulnérabilité à l'adaptation au changement climatique : éléments de réflexion pour les sciences sociales. In: Monaco A., Prouzet P. (dir.), Risques côtiers et adaptations des sociétés, ISTE Editions, 241-274.

Mahdhi N., Smida Z., Fouzai A., 2016. Farmers' strategies and determinants of choice to cope with the groundwater crisis in the private irrigated perimeters in south-eastern Tunisia. $2^{\text {nd }}$ EWaS International Conference, 1-4 June, 2016 - Chania, Crete, Greece.

MARE et GIZ (Ministère de l'Agriculture et de l'Environnement/GTZ, 2011. Elaboration de la "Stratégie Nationale sur le Changement Climatique de la Tunisie", Rapport de diagnostic. Version finale.

MEDD/DGEQV, 2008. Etude de la vulnérabilité environnementale et socio-économique du littoral tunisien face à une élévation accélérée du niveau de la mer due aux changements climatiques et de l'identification d'une stratégie d'adaptation. Rapport de synthèse. Version definitive, $57 \mathrm{pp}$.

MEE (Ministère de l'Equipement et de l'Environnement), 2013. Seconde Communication Nationale de la Tunisie à la Convention Cadre des Nations Unies sur les Changements Climatiques. http://mc3.lped. fr/spip.php?action=acceder_document\&arg=372\&$\mathrm{cle}=$

428d37c820702c1f27d4ab9852b57120689cc6f1\&file=pdf\%2Ftunnc2.pdf.

Moench M., 2007. When the well runs dry but livelihood continues: adaptive responses to groundwater depletion and strategies for mitigating the associated impacts. In Giordano M., Villholth K.G. (eds), The Agricultural Groundwater Revolution: Opportunities and Threats to Development, Oxford, UK: CABI Head Office.

Molle F., Venot J.Ph., Lannerstad M., Hoogesteger J., 2010. Villains or heroes? Farmers' adjustments to water scarcity. Irrigation and Drainage, 59: 419431.

Mwinjaka O., Gupta J., Bresser T., 2010. Adaptation strategies of the poorest farmers in drought-prone Gujarat. Climate and Development, 2(4): 346-363.

Nefzi A., 2012. Evaluation économique de l'impact du changement climatique sur l'agriculture : étude théorique et application au cas de la Tunisie. Thèse de doctorat, AgroParisTech. https://tel.archives-ouvertes.fr/pastel-01056525/.
ODS (Office de Développement de Sud), 2016. Médenine en chiffre, Ministère du Développement, de l'investissement et de la Coopération Internationale, Tunisie.

ODS (Office de Développement de Sud), 2016. Tataouine en chiffre, Ministère du Développement, de l'investissement et de la Coopération Internationale, Tunisie.

Omar A.R., Nawar M.H., El Bendary A.T., Abou Bakir H.E., 2015. Farms' perception of climate change in rural Damietta Governorate. Bull. Fac. Agric. Cairo Univ., 66: 1-9.

Paraïso A.A., Sossou G.C.A., Daouda I.Z.H., Yegbemey N.R., Sanni A., 2012. Perceptions and adaptations of beekeepers and honey hunters to climate change: The case of Natitingou and Tanguiéta in Northwest of Benin. African Crop Science Journal, 20: 523-532.

Pierre V.V., Rigobert C.T., Houinsou D., Hervé G., Olivier C.C., Simplice D.V., Euloge K., 2012. Perceptions et stratégies d'adaptation aux changements climatiques : le cas des communes d'Adjohoun et de Dangbo au Sud-Est Bénin. Les Cahiers d'Outre-Mer, 260: 479-492.

Seo N., Mendelsohn R., 2007. An analysis of crop choice: adapting to climate change in Latin American farms. World Bank Policy Research Working Paper 4162, Washington D.C.

Smit B., Skinner M.W., 2002. Adaptations options in agriculture to climate change: A typology. Mitigation and Adaptation Strategies for Global Change, 7: $85-114$.

Stéphane S., 2011. Adaptation au changement climatique dans le secteur de l'eau en Méditerranée : situation et perspectives. Les Cahiers du Plan Bleu, 10. http://planbleu.org/sites/default/files/publications/ cahier10_eaucc_fr.pdf.

Van Steenbergen F., Kaisarani A.B., Khan N.U., Gohar M.S., 2015. A case of groundwater depletion in Balochistan, Pakistan: enter into the voEndris GS, 2013. Understanding the process of adaptation to climate change by small-holder farmid. Journal of Hydrology: Regional Studies, 27: 36-47. http://dx.doi. org/10.1016/j.ejrh.2014.11.003.

Wheeler S., Zuo A., Bjornlund H., 2013. Farmers' climate change beliefs and adaptation strategies for a water scarce future in Australia. Global Environmental Change, 23(2): 537-547.

Yegbemey R.N., Yabi J.A., Aïhounton G.B., Paraïso A., 2014. Modélisation simultanée de la perception et de l'adaptation au changement climatique : cas des producteurs de maïs du Nord Bénin (Afrique de l'Ouest). Cahier d'agriculture, 23: 177-187. 